\title{
Plane Animation Simulation of the Interaction between Carbon Nanomaterials and Cell Lysosomes
}

\author{
Wen Liu $(\mathbb{D}$, Feng Qiu, and Xi Zeng \\ School of Art \& Design, Wuhan Institute of Technology, Wuhan 430205, Hubei, China \\ Correspondence should be addressed to Wen Liu; 09091401@wit.edu.cn
}

Received 4 August 2020; Revised 4 September 2020; Accepted 21 September 2020; Published 7 October 2020

Academic Editor: Tifeng Jiao

Copyright (c) 2020 Wen Liu et al. This is an open access article distributed under the Creative Commons Attribution License, which permits unrestricted use, distribution, and reproduction in any medium, provided the original work is properly cited.

\begin{abstract}
With the continuous development of cell dynamics, Raman scattering has become more and more common in the application of cell imaging and dynamic changes in chemical substances. This research mainly discusses the plane animation simulation process of the interaction between carbon nanomaterials and cell lysosomes. Twenty parallel HeLa cells were seeded on $40 \mathrm{~mm}$ imaging scaffolds. The $15 \%$ bovine placental serum cell culture cells are placed in a thermostat for 12 hours at a $\mathrm{CO}_{2}$ concentration of $6 \%$. After washing 4 times, $20 \mu \mathrm{L}$ of the dual control system is added to the confocal dish, and cycle optimization culture is performed at $2,4,6$, and 8 hours. It is incubated for 10 hours, 20 hours, and 30 hours $\left(35^{\circ} \mathrm{C}, 6 \% \mathrm{CO}_{2}\right)$. Next, the HeLa cells were taken out and seeded on three $30 \mathrm{~mm}$ confocal cell culture dishes. $\mathrm{CCl}_{4}$ is added to the initial confocal Petri dish. After heating for $30 \mathrm{minutes}$, the nanoparticle system is added to the two confocal Petri dishes and circulated within an appropriate time. After washing with PBS, the SERS signal in the cells was imaged with a laser confocal Raman microscope, and the excitation channels were GFP 475 and Cy 3 channels. LysoTrackerRed $(2 \mathrm{p} \mu \mathrm{M})$ was used for local experiments of cell isotope. Deoxygen $(2 \mathrm{p} \mu \mathrm{M})$ was used to induce cell death, and the $\mathrm{pH}$ changes in lysosomes in cells were imaged in real time with a confocal microscope. Two distinct peaks in the Raman spectrum were observed at $1246 \mathrm{~cm}^{-1}$ and $1543 \mathrm{~cm}^{-1}$. The research results show that the carbon nanomaterial synthesized by a simple method at room temperature has high stability and is suitable for analysis and detection of imaging with cells as the target.
\end{abstract}

\section{Introduction}

Lysosome damage may cause various diseases and is an important cause of tuberculosis, joint rheumatism, and other diseases. For the cause of silicosis, silica in the air is swallowed by phagocytic cells inside the body, and after fusion with lysosomes, silica can hydrogenate hydroxyl groups and membrane phospholipids or proteins in cell lysosomes. It will cause the internal environment of the cell to be disordered, the cell itself will be destroyed, the indigestible silica is released, and then, other neighboring cells will be destroyed. This repeated cycle is connected with cell damage and continuous repair and then further causes muscle fibrosis.

In the past, nonviral vectors contained a variety of inorganic nanomaterials. At present, gene carriers combining inorganic nanomaterials and cationic polymers have the size effect, the multifunctional properties of inorganic nanomaterials, and the strong gene loading capacity of cationic polymers. Based on this principle, in combination with cationic polymers, various nucleic acids can be effectively loaded so that carbon nanomaterials can better integrate into cell lysosomes without damaging the structure of cells. Therefore, studying the dynamic changes in carbon nanomaterials in cell lysosomes is more conducive for timely diagnosis of diseases.

The unique properties of carbon-based nanomaterials have aroused people's great interest, thereby promoting the development of large-scale industrial production methods. Zaytseva believes that, regardless of the application field, it is related to the increasing trend of the application of carbon nanomaterials to the environment, and it is still difficult to predict the biological impact in the environment. He researched different types of 
carbon-based nanomaterials, major production technologies, and important trends in agricultural and environmental applications. His research process is too simple and not specific enough [1]. Geng proposed a study to improve the morphology, separation performance, and mechanical properties of the membrane. The PVDF/MWCNT/GO hybrid membrane was successfully prepared by using polyvinylidene fluoride (PVDF) as the polymer as the nanofiller, the combination of the mixed matrix and the solution coagulation bath. The morphology and structure of MWCNT and GO were characterized, and the influence of the addition of carbon nanomaterials on the morphology, separation performance, and mechanical properties of the film was studied. His research process lacks data [2]. Smart fabrics and interactive textiles have aroused great interest as an emerging material due to their multifunctional functions. Lee manufactured a highly durable wireless flexible strain sensor based on commercial textiles through the integration of functional hybrid carbon nanomaterials and piezoresistive materials. Specifically, a solution-processable spray-assisted coating method capable of forming a uniform coating on a large area fabric is adopted. Due to their different deflection behaviors, textile-based strain sensors show highly stable and instant response in various bending curvatures and structural characteristics of $\mathrm{ZnO}$ nanowires. The accuracy of the wireless flexible strain sensor he studied is not enough [3]. Carbon nanomaterials (CNMs), such as carbon nanofibers (CNFs), multiwalled carbon nanotubes (MWCNTs), and carbon nanorods (CNRs) have various industrial and commercial applications. Angoth believes that exposure to these CNMs has also greatly increased. His research evaluated the extrapulmonary toxicity induced by these CNMs. After 1 day, 1 week, 1 month, and 3 months after exposure, the characteristics of CNM were tested by dripping into the rat trachea, by using serum biochemical parameters (such as alanine aminotransferase (ALT) and creatinine) and diagnostic assays kit to assess extrapulmonary toxicity. His research process lacks actual control [4]. Carbon nanomaterials (CNMs), including carbon nanotubes (CNTs) and their derivatives, have a variety of technical and biomedical applications. Woodman believes that the potential toxicity of CNM to cells and tissues has become an important emerging issue in nanotechnology. To evaluate the toxicity of carbon nanotubes and fullerenol $\mathrm{C}_{60}(\mathrm{OH})_{24}$, he used the budding yeast Saccharomyces cerevisiae, one of the simplest eukaryotes that shares the basic knowledge of eukaryotic cell biology. In order to study the underlying mechanism behind the growth defects induced by CNMs, he performed RNA-Seq-dependent transcription analysis and constructed a global gene expression profile of fullerenol $\mathrm{C}_{60}(\mathrm{OH})_{24}$ and $\mathrm{CNT}$-treated cells. His research process is too complicated [5].

This research mainly introduces the basic principles of the molecular dynamic method, establishes related models of carbon nanomaterials and cell lysosomes, and conducts preliminary simulations without external force control. A simple model (cell lysosome model) was established based on the characteristics of biofilms, and a pure single-walled carbon nanotube (carbon nanomaterial) was established. At the same time, a simulated environment model (water and saline model) was established. Based on the advantages of molecular dynamics and the characteristics of the model, corresponding simulation conditions are set. The effects of various spatial positions, various liquid environments (water environment and normal saline), and the size of carbon nanomaterials during the simulation are studied. These factors only have a slight influence on the interaction between carbon nanomaterials and cell lysosomes, but they did not cause direct contact between carbon nanomaterials and cell lysosomes.

\section{Nanomaterials}

2.1. Cell Lysosomes. Lysosomes have very important functions in cells. It can digest large particulate matter such as food and pathogenic bacteria into small molecules and can eliminate indigestible matter. The soluble enzymes of some aging cell organs and biological macromolecules can be transferred into the body, which is an important mechanism of body cell defense and self-replication. Therefore, lysosomes play a great role in the decomposition and digestion of cells.

Except for some cells such as red blood cells, most animal cells have lymphoid tissues, such as lysosomes, which play an important role in immune response, killing of harmful cells, and immune regulation. Cell lysosomes can kill pathogenic organisms such as bacteria, fungi, parasites, viruses, and aging cell organs that invade cells and play a very important role in the body's nonspecific immunity. Cell lysosomes can also digest microorganisms recognized by antibodies. However, cell lysosomes are also target cells that are often susceptible to H5N1 avian influenza virus. If the function of cell lysosomes to phagocytic cells is destroyed, it may prevent the $\mathrm{H} 5 \mathrm{~N} 1$ avian influenza virus from being excreted in the body and then further infects the patient with $\mathrm{H} 5 \mathrm{~N} 1$ avian influenza $[6,7]$.

2.2. Mechanical Behavior of Nanomaterials and Cell Membrane Coupling. The interaction between nanomaterials and cell membranes is accompanied by many complex biochemical processes. In recent years, researchers have gradually realized that external factors play an important role in the process of nanomaterials interacting with cell membranes, such as the human body's diet, internal circulation, and targeted transportation. These external factors include the following [8]:

(1) The size, shape, deformation stiffness, surface microstructure, and porosity of nanomaterials.

(2) The bending stiffness, surface tension, and initial structure of the cell membrane.

(3) The bonding of nanomaterials and cell membranes.

At different temperatures, nanomaterials exhibit different stress conditions: 


$$
\begin{aligned}
X\left(t-T_{1}\right)= & \left(\tau_{1} V_{C 1}+\tau_{2} V_{C 2}\right) \\
& -\left(\tau_{1} V_{C 1} e^{-\left(t-T_{1} / \tau_{1}\right)}+\tau_{2} V_{C 2} e^{-\left(t-T_{2} / \tau_{2}\right)}\right),
\end{aligned}
$$

where $V_{C 1}$ is the volume of nanomaterials and $\tau$ is the stress coefficient. Studies have shown that the deformable properties of nanomaterials have a great impact on the function of the immune system because cell lysosomes cannot swallow very soft targets. From the perspective of external factors, the interaction between nanomaterials and cell membranes includes the combination between the deformation of the cell membrane and the deformation of nanomaterials and the combination between the movement of biomolecules that drive encapsulation and encapsulation and many other chemical processes. The research on the combination of nanomaterial cell membranes and chemical behaviors aims to provide an understanding of the final form and related phenomena of nanomaterials through systematic and multiscale research [9].

The nanoparticles used have the same size and shape. In addition to disposing different ligands on the surface, they also cover the monolayer of ligands with the same hydrophobicity and durability ratio. Using a confocal microscope, the cell intake rate and intracellular distribution of fluorescently labeled nanoparticles in live mouse cells were measured. It was found that nanoparticles with alternating hydrophobic and hydrophilic ligands on the surface can penetrate the cell membrane without signs of cell membrane damage $[10,11]$. On the contrary, with the same hydrophobicity/hydrophilicity ratio, nanoparticles with hydrophobic and hydrophilic ligands randomly distributed on the surface will greatly reduce the possibility of penetrating the cell membrane. The terminal neurotic effect of the cell is blocked. It can be seen that the nanoparticles with rhombohedral ligands can directly invade the cell membrane, but the nanoparticles with random surface ligands cannot pass. Therefore, the distribution of ligands on the surface of nanoparticles plays a controlling role in the interaction between cells and nanoparticles [12].

\subsection{Medical Application Research of Fullerene Nanomaterials.} Many clinical and medical studies have reported the biological value of carbon nanomaterials, and its uses include DNA light splicing, enzyme blocking, tumor treatment, drug carriers, and antibacterial and cytoprotective effects $[13,14]$. Carbon nanomaterial fibers have been proven to be a promising technology for in situ structural health monitoring of polymer composites. Self-inductive composites can be achieved through a variety of integrated strategies, including carbon nanotube- (CNT-) coated fiber (CNTF), graphene oxide- (RGO-) coated fiber (RGOF), and carbon fiber (CF) [15]. RGOF has the highest track gauge sensitivity, with clear two-level performance from linear to nonlinear, and CNTF always shows a well-organized signal before the final fracture [16].

Due to the small size of carbon nanoparticles, they can reach lesions that cannot be reached by traditional drugs through the body. The superior hydrophobic part can help other substances passing through the cell membrane and can be used as a drug delivery carrier. For example, EPO is usually taken intravenously, but due to its sensitivity to intestinal enzymes, its activity will drop rapidly. When EPO is adsorbed by carbon nanomaterials, pharmacokinetic studies have shown that the biological activity of EPO is increased by 4 times $[17,18]$. Generally speaking, a nonlinear inhibition model is used to analyze the relationship between drug concentration and the effect of the target site in the body:

$$
E=E_{\max }-\left(E_{\max }-E_{\min }\right) \times \frac{C_{\text {eff }}^{s}}{E C_{50}^{s}+C_{\text {eff }}} .
$$

Among them, $E$ is the drug concentration in the blood and $C_{\text {eff }}^{s}$ is the drug concentration in the abdominal cavity [19].

On the one hand, carbon nanomaterials and their polypeptide derivatives because of the hydrophobic surface of the carbon nanomaterials exhibit antibacterial activity, are easy to penetrate into the cell membrane, and can destroy the lipids of the cell membrane. On the other hand, carbon nanomaterials can make pathogenic microorganisms inactive by hindering energy metabolism. It has been found that carbon nanomaterials and their derivatives can change the composition of HIV protease and hinder the activity of the enzyme, thereby achieving antiviral function. Coordination number refers to the calculation of the nearest atom number near the molecule, which can represent the distribution density around the atom to a certain extent [20]:

$$
g(r)=\frac{\mathrm{d} N}{4 \pi r^{2} \rho \mathrm{d} r}+\int \rho g(r) 4 \pi r^{2} \mathrm{~d} r+\rho \mathrm{d} r .
$$

Among them, $r$ is the central radius of the atom and $\mathrm{Nis}$ the number of atoms.

2.4. Raman Imaging. Raman spectroscopy is the basis of Raman spectroscopy imaging technology. It is the Raman scattering generated when light irradiates the material. The scattering types are mainly divided into two types: Rayleigh scattering and Raman scattering. Among the two types of scattering, Raman scattering becomes unstable because the material of a specific molecule is stimulated by light when it changes from the ground state to the unstable excited state [21]. The frequency components $f$ of the measured data of the dual-spectrum study have a phase combination relationship between $f z$ and $f+f$. In other words, they determine the second-order nonlinear relationship of the signal:

$$
\operatorname{TP}\left(f_{1}, f_{2}\right)=X_{j}\left(f_{1}\right) \cdot X_{j}\left(f_{2}\right) \cdot X_{j}\left(f_{1}+f_{2}\right) .
$$

In the formula, $X_{j}$ is the power spectrum.

Due to its high sensitivity, it can detect multiple targets at the same time, and the advantages of surface Raman enhancement such as fluorescence attenuation and light fading of the nonfluorescent probe are used in the design of a sensing system that detects multiple direct and indirect targets. The detection method is to directly combine the 
target object with the Raman base, which causes the change in the Raman characteristic peak $[22,23]$. Indirect detection refers to the interaction between the target molecule and the modified Raman matrix, but the Raman characteristic peak of the molecule has changed. Now, surface Raman enhancement is widely used in environmental testing of heavy carbons, polycyclic aromatic hydrocarbons, food additives, antibiotics, and residual pesticides, as well as biomedical testing [24].

\section{Plane Animation Simulation Experiment}

3.1. Obtaining and Processing the Initial Structure. Delete all unwanted compound molecules, leaving only the protein structure. The two models of carbon nanomaterial derivatives were established by Gaussview5.0 software, but the key length and key angle of this model may not be appropriate, so Gaussian09 software must be used to optimize its structure. HF/6-31G (d) was used to optimize the carbon nanomaterial derivative molecules, and the appropriate initial composition was obtained. The experimental equipment is shown in Table 1 .

3.2. Sample Processing. Plasma provided by volunteers is used in the laboratory. At the beginning of the experiment, $50 \mathrm{~mL}$ of blood was collected and made into erythrocyte hemolysis products. It is placed in a solution of $11 \%$ sodium chloride $(\mathrm{NaCl})$; then, the centrifuge tube is shaken well, diluted, and centrifuged for 15 minutes, and the blood sample is collected. Repeatedly, $50 \mathrm{ml}$ of ionized water is used three times. Under the action of osmotic pressure, the red blood cells rupture; the free hydroxy iron erythrocyte elements of the cells are freed and then centrifuged to remove the bottom of the tube membrane such as the sediment; and it is hemolyzed and stored in a sterilization tube at $4^{\circ} \mathrm{C}$. Before testing, the solution was diluted 50 times for analysis and testing of hemolytic products. In order to verify the feasibility of the established method, 10 blood samples were collected and centrifuged at $4000 \mathrm{rpm}$ for 40 minutes with an ultrafiltration tube (with a molecular weight of $4 \mathrm{KDa}$ ). $5 \mathrm{~mL}$ of centrifugal fluid is diluted with PBS buffer $(\mathrm{pH}=7)$ into $15 \mathrm{~mL}$ and stored in a refrigerator at $5^{\circ} \mathrm{C}$. In the process of glucose measurement, first $2 \mathrm{~mL}$ glucose standard solution (or blood sample) and $2 \mathrm{~mL}$ glucuronidase $(1 \mathrm{mg} / \mathrm{mL})$ are taken and then centrifuged in $10 \mathrm{~mL}$ PBS buffer solution for 20 minutes for chemiluminescence detection.

\subsection{Molecular Docking and Molecular Dynamic Simulation.} Before the docking process, the first receptor protein and ligand were processed using AutoDockTools in the next docking process. Using AutoDockVina software, two optimized carbon nanomaterial ligands were connected to the active center of PTP1B, and a reasonable initial complex structure was obtained. The receptor binding site is represented by a cube box, the center of the box is near the active center and the second binding site, and then, the box covers the residues of the entire active area. In the open and closed configuration, the three-dimensional grid points in the box are $30 \times 30 \times 30$ and $20 \times 20 \times 20$, respectively, and the interval between the grid points is set to $0.4 \mathrm{~A}$. The Monte Carlo method was used to retrieve the stereo conformation, and the energy scores of different docking stereo conformations were obtained. Finally, in the first 10 best conformations, considering the energy rating and the rationality of the conformation, the first complex that is most suitable for simulation is selected.

\subsection{Simulation of the Direct Penetration of Carbon Nano-} particles through the Film. In this study, carbon nanoparticles with a surface charge of $75 \%$ were initially selected. Through partial simulation, the free energy of the system is calculated by the umbrella sampling technique sampling method and weighted histogram analysis to obtain the average force potential (PMF) curve of the system and analyze the interaction between carbon nanoparticles and cell membranes. With carbon nanoparticles on the membrane (outside the cell), the free energy of the system decreases as the distance between the centers of gravity decreases. Therefore, carbon nanoparticles have a tendency to move from the outer membrane to the inner membrane, and the energy of the system is kept to a minimum. If carbon nanoparticles are placed under the membrane (inside the cell), the free energy of the system will increase as the distance between the centers of gravity decreases. That is, when carbon nanoparticles move into cells, an energy barrier is required. Therefore, if other substances in the cell are not involved in the uptake of carbon nanoparticles, the carbon nanoparticles will not enter the cytoplasm but stays inside the membrane. The attraction between the carbon nanoparticles and the bilayer membrane is mainly due to the electrostatic interaction between the cationic terminal of the ligand and the anion on the membrane. In addition, when the carbon nanoparticles are located on the lower side of the film, the generation value of the minimum energy point is the largest. This indicates that $75 \%$ of the surface-charged carbon nanoparticles penetrate the original plasma membrane and are embedded in it.

3.5. Confocal Inverted Raman Imaging. Twenty parallel HeLa cells were seeded on $2040 \mathrm{~mm}$ imaging scaffolds. The $15 \%$ bovine placental serum cell culture cells are placed in a thermostat for 12 hours at a $\mathrm{CO}_{2}$ concentration of $6 \%$. After washing 4 times, $20 \mu \mathrm{L}$ of the dual control system is added to the confocal dish, and cycle optimization culture is performed at 2, 4, 6, and 8 hours. Continue to incubate under the conditions of 10 hours, 20 hours, and 30 hours $\left(35^{\circ} \mathrm{C}, 6 \%\right.$ $\mathrm{CO}_{2}$ ). Next, HeLa cells were taken out and seeded on three $30 \mathrm{~mm}$ confocal cell culture dishes. $\mathrm{CCl}_{4}$ is added to the initial confocal dish. After heating for 30 minutes, the nanoparticle system is added to the two confocal dishes and circulated within an appropriate time. After washing with PBS, the SERS signal in the cells was imaged with a laser confocal inverted Raman microscope. The excitation channels were GFP 475 and Cy3 channels. LysoTrackerRed $(2 \mathrm{p} \mu \mathrm{M})$ was used for local experiments of cell isotope. 
TABle 1: Experimental equipment.

\begin{tabular}{lcc}
\hline Serial number & Equipment & Factory \\
\hline 1 & Fourier transform infrared spectrometer & Japan \\
2 & UV-visible spectrophotometer & USA \\
3 & JEM-2100 transmission electron microscope & Ray Magnetic Company \\
4 & Potential analyzer & American microphone \\
5 & X-ray diffractometer & Beijing Saiduo Company \\
\hline
\end{tabular}

Deoxygen $(2 \mathrm{p} \mu \mathrm{M})$ was used to induce cell death, and the $\mathrm{pH}$ changes in lysosomes in cells were imaged in real time with a confocal microscope.

3.6. Transmission Electron Microscope Test. The microstructure characteristics of the filled phase change material were studied, and the sample was tested by a transmission electron microscope (transmission electron microscope, TEM), model JEM-2100F. The suspension was prepared by mixing the sample in an ethanol solution after 15 seconds in ultrasonic waves, and the suspension was dropped into the porous copper mesh as a test sample.

\section{Raman Imaging Analysis}

4.1. In Vivo pH Imaging Analysis of Lysosome. In this study, a confocal microscope was used to observe the colocalization of ECDs and LysoTracker to investigate its distribution in cells. The results showed that ECDs showed bright green fluorescence in A549 cells or HEp-2 cells, and LysoTracker showed red fluorescence, and they coincided well. In order to further verify the lysosomal targeting performance of ECDs, ECDs were incubated for $12 \mathrm{~h}, 24 \mathrm{~h}$, or even $48 \mathrm{~h}$, and the fluorescence of ECDs and LysoTracker coincided to a large extent. The above experimental results show that ECDs can selectively target living cell lysosomes for a long time. In general, the prepared ECDs have high quantum yield (relative quantum yields $15 \%$, average value of three experiments), and excitation does not change with emission, good photobleaching resistance, good salt resistance, good oxidation resistance, and low toxicity. It has good biocompatibility and can indicate $\mathrm{pH}$ changes. Common interfering substances in cells do not interfere with $\mathrm{pH}$ response. At the same time, it has lysosomal targeting properties, so ECDs can be used to monitor the $\mathrm{pH}$ of lysosomes in the process of living cell apoptosis. The ECDs were incubated in A549 cells for 12 hours, and the fluorescence intensity changes in normal cells and apoptotic cells (with the addition of dexamethasone, an agent for inducing apoptosis) were observed with a fluorescent confocal microscope during 80 minutes. The table shows that the fluorescence intensity of normal cells is slightly reduced, while for apoptotic cells, the fluorescence of ECDs is significantly weakened due to the sudden increase in acidity. It is proved that the prepared emerald green fluorescent carbon dots can monitor the $\mathrm{pH}$ changes in the lysosome in real time. The analysis and detection method established in this experiment can successfully realize the determination of hemoglobin in normal human red blood cells, using the standard addition method (standard concentrations of hemoglobin are 1,5 , and $10 \mu \mathrm{M}$, respectively). Through the investigation of the recovery rate, the reproducibility and accuracy of the evaluation method are shown in Table 2 . The recovery rate of normal red blood cell samples randomly checked is in the range of $98.1 \%$ to $107.7 \%$. At the same time, the relative standard deviation (RSD) of each normal human red blood cell sample is lower than $2.3 \%$, indicating that the reproducibility and accuracy of this method are indeed very good. In short, the experiment proves that the established analytical detection method can successfully realize the highly selective detection of heme in normal human red blood cells.

4.2. Investigation and Analysis of Probe Dual Control Capability. The configuration changes in the system are composed of carbon nanoparticles with $75 \%$ positive charge on the surface and the film at different time periods, reflecting the dynamic process of carbon nanoparticles with a certain proportion of positive charge on the surface passing through the film. First, when the carbon nanoparticles are located on the outside of the membrane, they move rapidly towards the outer membrane, and the lipid molecules below also rapidly bulge upwards ( $0 \mathrm{~ns}-1.2 \mathrm{~ns}$ ). Then, it is continued to move downward, penetrating the outer film $(1.2 \mathrm{~ns}-20 \mathrm{~ns})$ at a speed lower than the initial speed. After that, it is embedded in the middle part of the membrane and gradually penetrates the inner membrane $(20 \mathrm{~ns}-30 \mathrm{~ns}$ ). Finally, it reaches the inner surface of the membrane and causes the inner membrane lipid molecules surrounding it to bulge, and the partial body is exposed to the intracellular environment (after $30 \mathrm{~ns}$ ). The Raman spectra of the probes in different control systems are shown in Figure 1. Obviously, the high speed at which the carbon nanoparticles approach the cell membrane in the first stage is the result of the rapid decrease in the free energy of the system due to the combined action of Coulomb force and van der waals force. Then, the nanoparticles are partially embedded in the outer film. At this time, the negative charge in the outer film reduces the rate of free energy decline, resulting in a lower speed in the second stage. In the third stage, the strong electrostatic interaction between the cation at the end of the ligand and the anion in the inner membrane promotes its continued movement into the cell. In the final stage, the interaction between the carbon nanoparticles and the film reaches an equilibrium state, which corresponds to the stable configuration obtained by the average force curve analysis. At this time, the free energy of the simulated system is the smallest. The synthesized probes were added to the buffer solution with $\mathrm{pH}=7.3$ without $\mathrm{ATP}, \mathrm{pH}=7.3$ with $10 \mu$ 
TABLE 2: Reproducibility and accuracy of the evaluation method.

\begin{tabular}{lccc}
\hline Samples & Added hematin $(\mu \mathrm{M})$ & Found $(\mu \mathrm{M})$ & Recovery $(\%)$ \\
\hline Human blood sample 1 & & & $106.0 \pm 0.8$ \\
1 & 1.0 & $1.061 \pm 0.05$ & $99.2 \pm 0.6$ \\
2 & 5.0 & $4.96 \pm 0.13$ & 1.7 \\
3 & 10.0 & $10.10 \pm 0.56$ & 1.3 \\
\hline Human blood sample 2 & 1.0 & & \\
4 & 5.0 & $1.07 \pm 0.08$ & $107.0 \pm 0.6$ \\
5 & 10.0 & $5.21 \pm 0.21$ & $104.2 \pm 0.3$ \\
6 & $10.21 \pm 0.43$ & $102.1 \pm 1.1$ & 1.1 \\
\hline
\end{tabular}

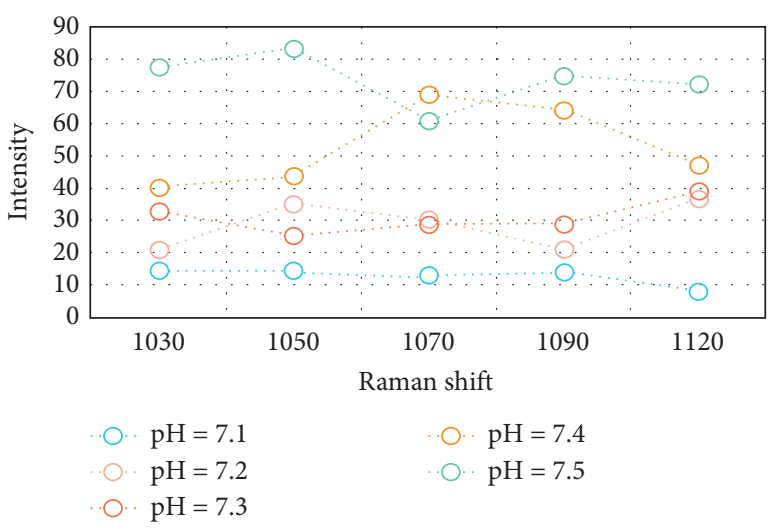

Figure 1: Raman spectra of probes in different control systems.

MATP, $\mathrm{pH}=3.5$ without $\mathrm{ATP}$, and $\mathrm{pH}=3.5$ with $10 \mu$ MATP. After reacting for 5 minutes, $10 \mu \mathrm{L}$ of the solution was dropped on the surface of the silicon wafer to form small droplets, and the SERS signal was collected under laser irradiation. It can be seen from the Raman spectrum that, in a neutral environment, the Raman signal is very weak regardless of the presence of ATP. Under acidic conditions, there will be some background signals when there is no ATP. It may be that some connected DNAs do not form a double in the acidic chain, and the configuration of $\mathrm{i}$-motif changes and generates a certain Raman signal, but it can be used as a background. Under acidic conditions, when there is ATP, the Raman signal is significantly enhanced. The chain double-control structure and the double-control system in which nanoparticles aggregate to form Raman hot spots can image ATP in the lysosome.

4.3. Surface Charge Density of Carbon Nanoparticles. Carbon nanoparticles with 20\%, 40\%, 60\%, 80\%, and $100 \%$ positive charges on the surface were constructed to study the effects of carbon nanoparticles with different surface charge densities on their interaction with cell membranes. Figure 2 shows the results after 100 ns of interaction between carbon nanoparticles with different charged ratios and real cell membrane models. Obviously, carbon nanoparticles with $0 \%$ charge on the surface have no driving force to move towards the membrane due to their neutral and hydrophobic properties, and they are not close to the cell membrane. For charged carbon nanoparticles, as the proportion of surface charge increases, the degree of penetration of carbon nanoparticles through the film has been deepened; when the

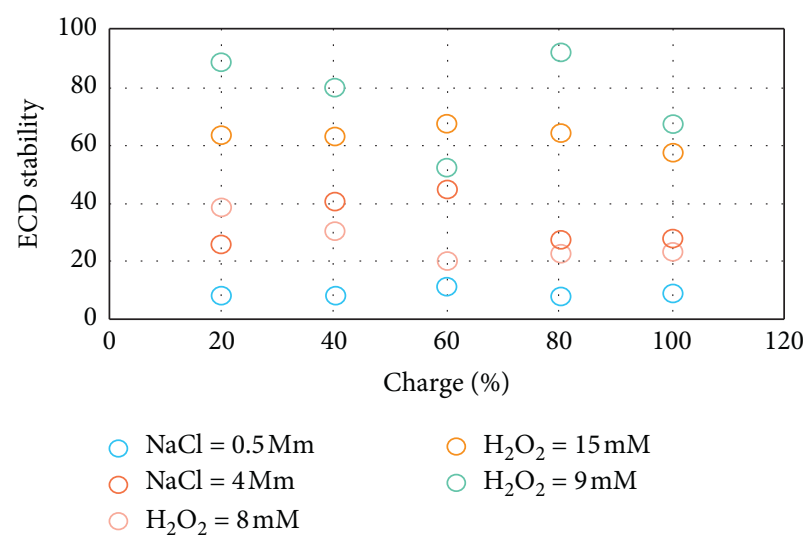

FIgURE 2: Results of 100 ns interaction between carbon nanoparticles with different charge ratios and the real cell membrane model ECDs stability.

proportion of surface charge is less than $60 \%$, the carbon nanoparticles are only embedded in the middle of the film. Because when the limited electric field force pulls the carbon nanoparticles to the hydrophobic region of the lipid molecules, the hydrophobicity of the carbon nanoparticles and the van der waals force between them and the membrane hinder them from continuing to penetrate the membrane. For carbon nanoparticles with $80 \%$ positive charge on the surface, they penetrate the cell membrane and cause the inner membrane around them to form a bump. It is continued to increase the charged ratio (100\%). After the carbon nanoparticle penetrates the membrane, it is almost completely wrapped by lipid molecules, forming a structure similar to a vesicle. This phenomenon is caused by the excess between the end of the ligand and the inner membrane, showing strong electrostatic attraction. This paper also studied the cytotoxicity of carbon nanoparticles during ingestion. First, the average root variance of the cell membrane is calculated relative to its initial structure after the carbon nanoparticles penetrate the membrane. Its value can reflect the degree of structural change in the membrane. The larger the value, the more obvious the membrane change. It can be seen that, as the surface charge ratio increases, the overall trend of the mean root variance value is also increasing; that is to say, the surface charge ratio of carbon nanoparticles has a great influence on the integrity of the cell membrane. With ECDs' salt resistance and antioxidant capacity, no matter when the concentration of sodium chloride is $0.5-4 \mathrm{Mm}$ or the concentration of hydrogen 
peroxide is $8-15 \mathrm{mM}$, the fluorescence intensity of ECDs has not changed significantly, indicating the carbon dot. It has good salt resistance and antioxidant capacity. In short, ECDs synthesized by a simple method at room temperature have good stability and are very suitable for analysis and detection and cell-targeted imaging.

Then, the total leakage of water molecules in the process of penetrating the membrane is calculated, that is, the number of water molecules that enter the cytoplasm from the outside of the cell membrane. From the above simulation, it can be found that the carbon nanoparticles will cause a certain degree of damage to the cell membrane structure during the process of penetrating the membrane, so the solvent outside the membrane can easily enter the inside of the membrane. Figure 3 shows the variation in the leakage of water molecules with the charge. And because human cells are a relatively independent and balanced system, any substances outside the balance flowing into the cell may cause the acid-base balance inside and outside the cell to be broken, leading to functional damage and even cell death, so the amount of water leakage is measured: The cytotoxicity of carbon nanoparticles is a more direct aspect. It can be seen in Figure 3 that as the proportion of charged electricity increases, the amount of water leakage first increases and then gradually decreases after approximately $60 \%$ reaches the maximum value. This overall trend is consistent with the value of the centroid distance between the carbon nanoparticles and the film when it is finally stable and also proves the correctness of the calculation of the water leakage program in this study. Based on the above analysis of the cytotoxicity of carbon nanoparticles with different charged ratios, it can be seen that when the charged ratios are $20 \%$ and $60 \%$, the damage to the membrane is the smallest and the largest. However, in the case of $20 \%$ charged ratios, due to a small electrostatic attraction, the carbon nanoparticles did not pass through the cell membrane. In addition, considering the release process of carbon nanoparticles after penetration, carbon nanoparticles with about $80 \%$ of the surface will be the best choice for drug delivery due to their lower cytotoxicity and higher penetration efficiency.

4.4. Analysis of Carbon Spots in Lysosomes. Subsequently, the carbon dots synthesized by a simple method in this article were characterized. The carbon dots are quasispherical with uniform particle size distribution. 100 particles are randomly counted, and the average particle diameter of the carbon dots is $11.8 \mathrm{~nm}$. The lattice distribution of carbon dots is obvious, and the lattice parameters are $0.21 \mathrm{~nm}$ and $0.31 \mathrm{~nm}$, respectively, which correspond to the 100 planes 188 in the graphene plane and the 002 plane between the graphene planes. The prepared carbon dots have an obvious ultraviolet absorption peak at $282 \mathrm{~nm}$, which is attributed to the $\pi-\pi$ electronic transition of $\mathrm{C} \_\mathrm{C} / \mathrm{C}=\mathrm{C}$. At the same time, the carbon dots show the property that emission depends on excitation, and its maximum excitation wavelength is $350 \mathrm{~nm}$ and the maximum emission wavelength at $460 \mathrm{~nm}$; under the excitation of $365 \mathrm{~nm}$ ultraviolet light, it shows bright blue fluorescence. In addition, we also investigated

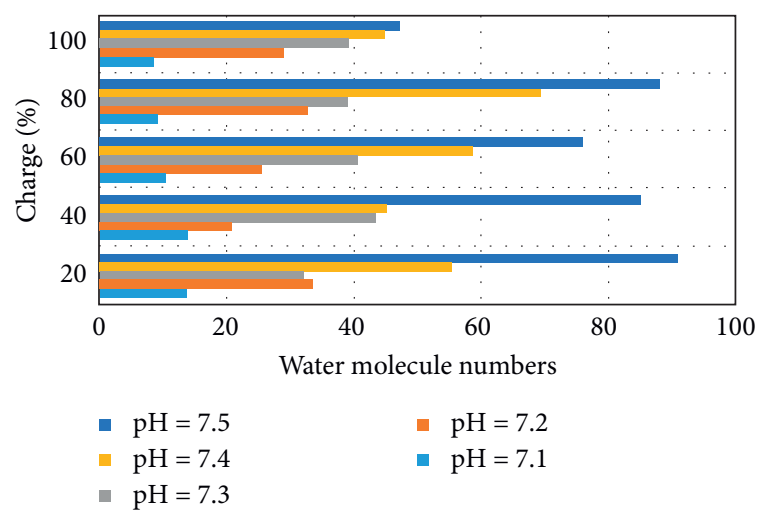

FIGURE 3: The number of leaked water molecules varies with charge.

the cytotoxicity of carbon dots. Even if the concentration of carbon dots is as high as $0.01 \mathrm{mg} / \mathrm{mL}$, human laryngeal carcinoma epithelial cells (HEp-2 cells) still have a survival rate of more than $85 \%$, indicating that the prepared carbon dots have good biocompatibility, and carbon dots are mostly distributed in the cytoplasm, almost not entering the nucleus, which is consistent with the carbon dots reported in most literature. The carbon dots corresponding to different covalent bonds are shown in Figure 4. The results are as follows: X-ray electron spectroscopy (XPS) shows that the carbon dots are mainly composed of carbon, oxygen, and nitrogen. The peaks corresponding to the XPS spectrum are as follows: $284.8 \mathrm{eV}, 399.3 \mathrm{eV}$, and $533.1 \mathrm{eV}$, and the element content is $71.05 \%, 25.2 \%$, and $3.75 \%$; these elements just correspond to the elements in the raw materials. After analyzing the high-resolution XPS, the carbon dots have the following bonds: C-C, C-N, C=O, C_OH, and C-N_C. In order to further analyze the distribution of the surface functional groups of the carbon dots, the carbon dots were analyzed by FTIR, and the infrared absorption peak of $3448 \mathrm{~cm}^{-1}$ corresponds to the stretching vibration peak of $\mathrm{NH} / \mathrm{OH}$, and the peak of $2920 / 2854 \mathrm{~cm}^{-1}$ corresponds to the stretching vibration peak of the $\mathrm{CH}$ bond, while the infrared absorption peaks of 1641 and $1083 \mathrm{~cm}^{-1}$ correspond to the stretching vibration of $\mathrm{C}=\mathrm{O}$ and $\mathrm{CO}$. Together with the results of FTIR, it proved that the surface of carbon dots has abundant functional groups such as amino, carboxyl, and hydroxyl groups, which makes the carbon dots have good water solubility. Finally, the Raman spectroscopy analysis of the synthesized carbon dots shows that there are two obvious peaks in the Raman spectra, located at $1246 \mathrm{~cm}^{-1}$ and $1543 \mathrm{~cm}^{-1}$. The former belongs to the D-band peak of $\mathrm{sp}$ carbon, and the latter belongs to the G-band peak of $\mathrm{sp}^{2}$ carbon, which is consistent with the previous HRTEM results, which proves that the carbon dots have both $\mathrm{sp}^{2}$ and $\mathrm{sp}^{3}$ hybrid carbons. In summary, the above results show that, by selecting simple and easily available raw materials, a onestep solvothermal method has synthesized excellent carbon dots with excellent properties, abundant water-soluble functional groups, and good biocompatibility, which can be used for analysis, detection, and cell imaging. Due to the mild reaction conditions, the abundant amino functional 


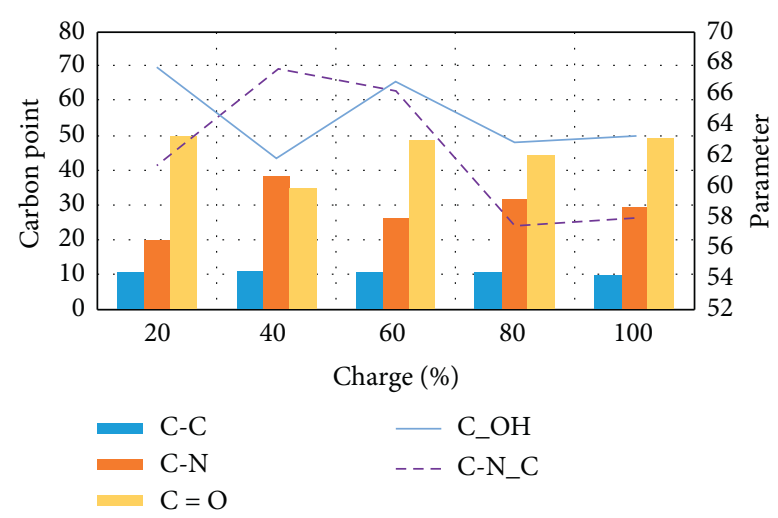

FIgURE 4: Carbon dots corresponding to different covalent bonds.

groups in the raw materials still remain in the ECDs, and the abundant amino, carboxyl, and hydroxyl functional groups on the surface of the ECDs endow ECDs with good water solubility, which is the carbon point as a $\mathrm{pH}$ response probe and intracellular solution. Enzyme targeting probes provide the possibility.

\section{Conclusion}

According to research, nanoparticles not only damage animal tissues and organs but also may show toxicity to various degrees from cells to protein genetic factors. The toxicity mechanism of nanoparticles has not yet been determined. C60 can cause the generation of reactive oxygen species (ROS), which may cause damage to cell membranes and cytotoxicity. Therefore, if nanomaterials are widely used, evaluating their safety is an urgent issue. It is necessary to conduct detailed research on the role and toxicity mechanism of nanometers to improve the biological safety of carbon nanomaterials.

In recent years, as a single-layer two-dimensional carbon nanomaterial, carbon nanomaterials have made great progress in the field of biomedicine. Due to their unique physical and chemical properties such as excellent biocompatibility and changeability, they have made greater progress. It can also participate in drug transport and photothermal effects in organisms. In this paper, a functional oxide nanomaterial is designed and synthesized, and its characteristics are changed to have a larger specific surface area, aromatic molecule loading capacity, conjugation, and a compound that can interact with hydrogen bonds and apply it to the treatment of chemical photothermal reaction and gene transfer. The results show that the synthesized carbon nanomaterials have great potential applications in the field of biomedicine.

Now, carbon nanotubes are widely used, and they are being used more and more in the field of biology. In the research direction of carbon nanotubes, or in the research direction of biological toxicity, whether they function as drugs or gene carriers must first be confirmed and then whether the specific mechanism of carbon nanotubes is a common problem. Carbon nanotubes enter the cell body, so the interaction between carbon nanotubes and biofilms is a study of carbon nanotubes in the biomedical field. The hydrophilic and hydrophobic carbon nanomaterials prepared in this study show excellent application prospects in biological imaging or biomarker research. Hydrophobic carbon nanomaterials easily enter cells, accumulate in cells, and emit bright red fluorescence. Therefore, this research provides a new method for the preparation and development of carbon nanomaterials for the study of biological imaging.

\section{Data Availability}

The data used to support the findings of this study are available from the corresponding author upon request.

\section{Conflicts of Interest}

The authors declare that they have no conflicts of interest.

\section{Acknowledgments}

This work was supported by the Youth Project Fund for Humanities and Social Sciences Research of Hubei Education Department (17Q080).

\section{References}

[1] O. Zaytseva and G. Neumann, "Carbon nanomaterials: production, impact on plant development, agricultural and environmental applications," Chemical \& Biological Technologies in Agriculture, vol. 3, no. 1, p. 17, 2016.

[2] H. Z. Geng, C. X. Xu, W. Y. Wang et al., "Preparation and performance of carbon nanomaterials/polyvinylidene fluoride hybrid membrane," Journal of Tianjin Polytechnic University, vol. 37, no. 4, pp. 1-6, 2018.

[3] T. Lee, W. Lee, .S.-W. Kim, J. J. Kim, and B.-S. Kim, "Flexible textile strain wireless sensor functionalized with hybrid carbon nanomaterials supported $\mathrm{ZnO}$ nanowires with controlled aspect ratio," Advanced Functional Materials, vol. 26, no. 34, pp. 6206-6214, 2016.

[4] B. Angoth, N. R. H. Lingabathula, and N. R. Yellu, "Assessment of extrapulmonary toxicity induced by carbon nanomaterials following intra-tracheal instillation in rats," Asian Journal of Pharmaceutical and Clinical Research, vol. 10, no. 5, p. 82, 2017.

[5] S. Woodman, H. Short, A. K. Linan et al., "Carbon nanomaterials alter global gene expression profiles," Journal of Nanoscience and Nanotechnology, vol. 16, no. 5, pp. 52075217, 2016.

[6] G. Wang, Y. Wang, P. Zhang et al., "Structure dependent properties of carbon nanomaterials enabled fiber sensors for in situ monitoring of composites," Composite Structures, vol. 195, pp. 36-44, 2018.

[7] A. N. Li, Y. V. Plekhanova, S. E. Tarasov et al., "Effect of some carbon nanomaterials on ethanol oxidation by gluconobacter oxydans bacterial cells," Applied Biochemistry and Microbiology, vol. 53, no. 1, pp. 123-129, 2017.

[8] M. Zhou, H. L. Wang, and S. Guo, "Towards high-efficiency nanoelectrocatalysts for oxygen reduction through engineering advanced carbon nanomaterials," Chemical Society Reviews, vol. 47, no. 16, pp. 1273-1307, 2016.

[9] L. Cui, X. Y. Zhang, and G. Wei, "Computational study on thermal conductivity of defective carbon nanomaterials: 
carbon nanotubes versus graphene nanoribbons," Journal of Materials Science, vol. 53, no. 6, pp. 4242-4251, 2018.

[10] S. Araby, R. A. Qiu, R. Wang, Z. Zhao, C.-H. Wang, and J. Ma, "Aerogels based on carbon nanomaterials," Journal of Materials Science, vol. 51, no. 20, pp. 9157-9189, 2016.

[11] W. J. Ma, J. Lim, and S. O. Kim, "Nitrogen dopants in carbon nanomaterials: defects or a new opportunity?" Small Methods, vol. 1, no. 1-2, p. 1600014, 2017.

[12] J. Majewska and B. Michalkiewicz, "Preparation of carbon nanomaterials over Ni/ZSM-5 catalyst using simplex method algorithm," Acta Physica Polonica A, vol. 129, no. 1, pp. 153-157, 2016.

[13] A. Hajian, S. B. Lindström, T. Pettersson, M. M. Hamedi, and L. Wågberg, "Understanding the dispersive action of nanocellulose for carbon nanomaterials," Nano Letters, vol. 17, no. 3, p. 1439, 2017.

[14] D. A. Wågberg, M. I. Tulepov, S. Tursynbek et al., "Processing of the bottomhole zones of oil wells with use of the carbon nanomaterials," Rasayan Journal of Chemistry, vol. 10, no. 2, pp. 344-348, 2017.

[15] E. X. Ding, H. Z. Geng, J. Wang et al., "Hierarchical chrysanthemum-flower-like carbon nanomaterials grown by chemical vapor deposition," Nanotechnology, vol. 27, no. 8, Article ID 085602, 2016.

[16] K. Ozdemir, "Experimental investigation of trihalomethanes removal in chlorinated drinking water sources with carbon nanomaterials," Fresenius Environmental Bulletin, vol. 25, no. 12, pp. 6202-6214, 2016.

[17] P. Choudhury, S. D. Mandal, and P. K. Das, "Hydrophobic end-modulated amino-acid-based neutral hydrogelators: structure-specific inclusion of carbon nanomaterials," Chemistry - A European Journal, vol. 22, no. 15, pp. 51605172, 2016.

[18] D. Veclani, A. M. Tolazzi, and A. Melchior, "Molecular interpretation of pharmaceuticals' adsorption on carbon nanomaterials: theory meets experiments," Processes, vol. 8, no. 6, p. 642, 2020.

[19] S. Liu, C. Ma, M.-G. Ma, and J.-F. Li, "Recent advances in carbon nanomaterials derived from biomass," Science of Advanced Materials, vol. 11, no. 1, pp. 5-17, 2019.

[20] S. Eissa, M. Almusharraf, and M. Zourob, "A comparison of the performance of voltammetric aptasensors for glycated haemoglobin on different carbon nanomaterials-modified screen printed electrodes," Materials Science and Engineering: C, vol. 101, pp. 423-430, 2019.

[21] M. Du, Y. Gao, H. Su, and H. Fang, "Carbon nanomaterials enhanced cement-based composites: advances and challenges," Nanotechnology Reviews, vol. 9, no. 1, pp. 115-135, 2020.

[22] Y. Jiang, X. L. Zhang, and T. Zhang, "Improving the performance of UV-curable coatings with carbon nanomaterials," Express Polymer Letters, vol. 12, no. 7, pp. 628-639, 2018.

[23] M. R. Taha and J. M. A. Alsharef, "Performance of soil stabilized with carbon nanomaterials," Chemical Engineering Transactions, vol. 63, no. 2018, pp. 757-762, 2018.

[24] F. Yu, B. Z. Chen, B. Wang, Z. Jin, S. Ma, and X. Liu, "The role of lysosome in cell death regulation," Tumor Biology, vol. 37, no. 2, pp. 1427-1436, 2016. 